\title{
Time determines the neural circuit underlying associative fear learning
}

\section{Marta Guimarãis, Ana Gregório, Andreia Cruz, Nicolas Guyon and Marta A. Moita*}

Fundação Champalimaud Neuroscience Program, Instituto Gulbenkian de Ciência, Oeiras, Portugal

\section{Edited by:}

Nora Abrous, Institut des

Neurosciences de Bordeaux, France

\section{Reviewed by:}

Valérie Doyère, CNRS, France

Aline Desmedt, Université

Bordeaux 1, France

*Correspondence:

Marta A. Moita, Fundação

Champalimaud Neuroscience

Program, Instituto Gulbenkian de

Ciência, Rua da Quinta Grande 6,

2780-156 Oeiras, Portugal.

e-mail:marta.moita@neuro.

fchampalimaud.org

\begin{abstract}
Ultimately associative learning is a function of the temporal features and relationships between experienced stimuli. Nevertheless how time affects the neural circuit underlying this form of learning remains largely unknown. To address this issue, we used single-trial auditory trace fear conditioning and varied the length of the interval between tone and footshock. Through temporary inactivation of the amygdala, medial prefrontal-cortex (mPFC), and dorsal-hippocampus in rats, we tested the hypothesis that different temporal intervals between the tone and the shock influence the neuronal structures necessary for learning. With this study we provide the first experimental evidence showing that temporarily inactivating the amygdala before training impairs auditory fear learning when there is a temporal gap between the tone and the shock. Moreover, imposing a short interval ( $5 \mathrm{~s}$ ) between the two stimuli also relies on the mPFC, while learning the association across a longer interval $(40 \mathrm{~s})$ becomes additionally dependent on a third structure, the dorsalhippocampus. Thus, our results suggest that increasing the interval length between tone and shock leads to the involvement of an increasing number of brain areas in order for the association between the two stimuli to be acquired normally. These findings demonstrate that the temporal relationship between events is a key factor in determining the neuronal mechanisms underlying associative fear learning.
\end{abstract}

Keywords: amygdala, mPFC, hippocampus, trace fear conditioning, muscimol, single-trial

\section{INTRODUCTION}

The mammalian brain is able to associate events across a broad range of time intervals, from millisecond (Christian and Thompson, 2003; Woodruff-Pak and Disterhoft, 2008) to hours (Welzl et al., 2001; Stehberg and Simon, 2011). The association of two stimuli separated in time depends on the persistence in the brain of a mnemonic "trace" of the first stimulus until the subsequent one occurs. How the temporal distance between the events influences the neuronal mechanisms required for such persistence remains unknown.

In Pavlovian fear conditioning, extensively used to study associative learning, animals learn to fear a neutral stimulus, such as a tone, after it is paired with an aversive foot-shock (LeDoux, 2003; Maren, 2008). In auditory trace fear conditioning (tFC), the two stimuli are separated in time. In this paradigm tone-shock association has been shown to occur across different trace intervals lengths, from 0.5 to $45 \mathrm{~s}$ (Baeg et al., 2001; Quinn et al., 2002; Misane et al., 2005), being acquired after a single-trial (Stiedl and Spiess, 1997; Misane et al., 2005). Thus, this paradigm is suited to investigate how differences in the temporal distance between two stimuli may influence the mechanisms underlying associative learning.

Both medial prefrontal-cortex (mPFC) and the hippocampus have been implicated in tFC. Consistent with the involvement of mPFC in tasks which require processing of temporally disconnected stimuli (Fuster et al., 2000) both pharmacological and electrophysiological studies have shown that $\mathrm{mPFC}$ is involved in $\mathrm{tFC}$ (Baeg et al., 2001; Runyan et al., 2004; Gilmartin and McEchron, 2005a; Gilmartin and Helmstetter, 2010). In addition, numerous studies using lesion (McEchron et al., 1998), pharmacological and genetic manipulations (Huerta et al., 2000; Chowdhury et al., 2005; Quinn et al., 2005), and single-unit recordings (McEchron et al., 2003; Gilmartin and McEchron, 2005b) implicated the dorsal-hippocampus in tFC. Hence, these two structures have been postulated to maintain information about the tone until shock delivery. Importantly, both these areas are reciprocally connected with the amygdala (McDonald, 1998; Pitkänen et al., 2000; Hoover and Vertes, 2007). Under conditions in which tone and shock are contiguous as in auditory delay fear conditioning (dFC), several studies using electrophysiological recordings, discrete lesions, temporary inactivation, genetic, and pharmacological manipulations have consistently demonstrated that the amygdala plays a crucial role in associative fear learning (Fanselow and LeDoux, 1999; LeDoux, 2003; Rumpel et al., 2005; Han et al., 2007, 2009; Maren, 2008; Ciocchi et al., 2010; Haubensak et al., 2010; Pape and Pare, 2010). Therefore, it is generally accepted that tFC relies on the amygdala with an fMRI study in humans supporting this assumption (Büchel et al., 1999). Nevertheless, a recent study done in mice reported that tFC could be acquired independently of the amygdala (Raybuck and Lattal, 2011). Therefore, the role of the amygdala in tFC needs further investigation ${ }^{1}$.

\footnotetext{
${ }^{1}$ Meanwhile a study using protein synthesis blockade in the rat amygdala showed that this structure is critical for normal memory consolidation both in delay and trace fear conditioning (Kwapis et al., 2011).
} 
Recently, through the use of post-training hippocampal lesion or the injection of NMDA receptor antagonist, it was shown that the dorsal-hippocampus is involved in tFC only when trace intervals longer than $10 \mathrm{~s}$ are used (Chowdhury et al., 2005; Misane et al., 2005). This suggests that hippocampal plasticity during learning and an intact hippocampus during recall are important when long intervals separate tone and shock, however it remained to be established whether hippocampal activity during learning followed the same temporal profile. In addition, this studies raised the question of what structures are involved when shorter intervals are used and how they differ from the ones required for dFC, where no interval exists separating the two stimuli.

We addressed these questions by assessing the involvement of the amygdala, the mPFC, and the dorsal-hippocampus in $\mathrm{tFC}$ acquisition when either a short or a longer trace interval is imposed between the tone and the shock. To this end, muscimol (a GABA receptor agonist) was used to temporarily inactivate each one of these structures prior to a short tFC protocol (short tFC), where tone and shock were separated by $5 \mathrm{~s}$, or prior a long tFC protocol (long tFC) where the two stimuli were separated by a longer interval lasting $40 \mathrm{~s}$. The involvement of each structure in the acquisition of the short $\mathrm{tFC}$ or the long $\mathrm{tFC}$ was examined by assessing differences in the levels of fear displayed to the tone.

\section{RESULTS}

In this study we performed three different experiments. In all three conditions infusions were done immediately prior to training. A group of rats were infused with muscimol $(1.6 \mathrm{mM})$ to block neural activity and a control group with the vehicle solution (PBS). Infusions were targeted to the basolateral complex of the amygdala (Experiment 1 and 2), the dorsal-hippocampus (Experiment 1 and 2) or to the pre-limbic region of mPFC (Experiment 1,2 , and 3). Rats were then assigned to a training protocol where the interval between the tone and shock was either short $=5 \mathrm{~s}$ (Experiment 1), long $=40 \mathrm{~s}($ Experiment 2$)$, or non-existent $=0 \mathrm{~s}$ (Experiment 3). Importantly, in all three experiments the training protocol consisted of a single tone-shock pairing to insure that possible differences in the neural circuit underlying learning in each one of the protocols were not due to variations in the inter-trial/inter-stimuli-interval ratio (Gallistel and Gibbon, 2000; Balsam and Gallistel, 2009). To assess the effect of inactivation during training in all three experiments rats were tested drug free in the following day for fear to the tone. The level of freezing response was used as the learning index (see Figure 1).

\section{EXPERIMENT 1}

Role of the amygdala, mPFC, and dorsal-hippocampus in the acquisition of short tFC (tone and shock separated by $5 \mathrm{~s}$ ).

The first experiment accessed the involvement of the amygdala, the $\mathrm{mPFC}$, or the dorsal-hippocampus in the acquisition of auditory fear conditioning when a short trace interval is used (tone and shock separated by $5 \mathrm{~s}$ ). Comparisons were done between rats that received pre-training muscimol or vehicle infusions (PBS) into the amygdala (amyg-mus $n=9$; amy-veh $n=8$ ), the mPFC (mPFC-mus $n=7$; mPFC-veh $n=9$ ), or the dorsal-hippocampus (dHipp-mus $n=7$; dHipp-veh $n=6$ ). The training protocol consisted of one-trial in which a 20-s tone was followed by short interval ( $5 \mathrm{~s})$ at the end of which a shock $(1.5 \mathrm{~mA} ; 1.5 \mathrm{~s})$ was delivered. To assess the acquisition of fear to the tone three tones $(20 \mathrm{~s}$ each) were delivered during the tone test session performed $24 \mathrm{~h}$ later when animals were drug free. For each animal a freezing score was calculated as the average freezing across the three tones. A twoway ANOVA, testing for main effect of structure (amygdala, mPFC, or dorsal-hippocampus) and treatment (vehicle or muscimol), showed a significant effect of structure $\left(F_{2,40}=3.73, P=0.032\right)$, a significant effect of treatment $\left(F_{1,40}=27.47, P<0.001\right)$ and a significant interaction $\left(F_{2,40}=3.26, P=0.048\right)$. LSD post hoc analysis Bonferroni corrected for multiple comparisons $(\alpha=0.017)$ showed that rats with the amygdala inactivated before conditioning froze significantly less to the tone than vehicle infused rats $(P<0.001)$. Similarly, mPFC-muscimol rats displayed significantly less freezing compared to vehicle controls $(P=0.005)$. In contrast, no significant difference was found between treatments in the dorsal-hippocampus with both muscimol and vehicle groups displaying high levels of freezing to the tone ( $P=0.235$; Figure 2$)$. This finding is in agreement with previous studies showing that the dorsal-hippocampus does not significantly contribute to auditory $\mathrm{tFC}$ when intervals shorter than $10 \mathrm{~s}$ are used (Misane et al., 2005).

To examine whether the effects of amygdala and mPFC inactivation on tone-evoked freezing could be explained by differences in baseline fear, we measured freezing during the 20-s that immediately preceded the first tone (pre-CS freezing). A Kruskal-Wallis test comparing pre-CS freezing across all groups revealed a significant effect $\left[H_{(5,46)}=12.6, P=0.02\right]$. Post hoc pairwise comparisons (Bonferroni corrected) revealed that the $\mathrm{mPFC}$-muscimol group was significantly different from the BLA-vehicle group and that no other comparison yielded a significant difference. Importantly, no difference in pre-CS freezing between $\mathrm{mPFC}$-vehicle and mPFC-muscimol or BLA-vehicle and BLA-muscimol were found. Therefore, baseline freezing levels do not explain the differences in freezing during the tone observed between muscimol and vehicle groups of amygdala and mPFC cannulated animals. Hence, our results demonstrate that normal neuronal activity, both in the amygdala and in the mPFC, is important for normal acquisition of tone-shock association across a short trace interval (5s).

As the amygdala has been shown to respond to shock USs and inactivating this structure has been shown to affect US responses (Blair et al., 2005; Johansen et al., 2010), we compared post-shock freezing of rats infused with vehicle or muscimol in the amygdala and found no difference (veh $=15.0 \pm 3.7$, mus $=14.3 \pm 7.5$ ). In addition, visual inspection of shock reactivity, such as running and jumping, suggested no difference between the two groups. Thus, the effect of amygdala inactivation on freezing to the tone is better explained by an effect on learning than impaired shock processing.

Finally, we tested learned fear of the training environment and found that this experimental protocol did not induce reliable contextual fear learning, such that in all vehicle groups there were rats that displayed freezing levels below $10 \%$. Nonetheless, in accordance we previous studies (Muller et al., 1997; Esclassan et al., 2009; Gilmartin and Helmstetter, 2010), we found that muscimol infusion in all three structure tended to yield lower freezing levels compared to vehicle infusion (amygdala: $v e h=31.3 \pm 4.1$, mus $=1.6 \pm 0.5$; 

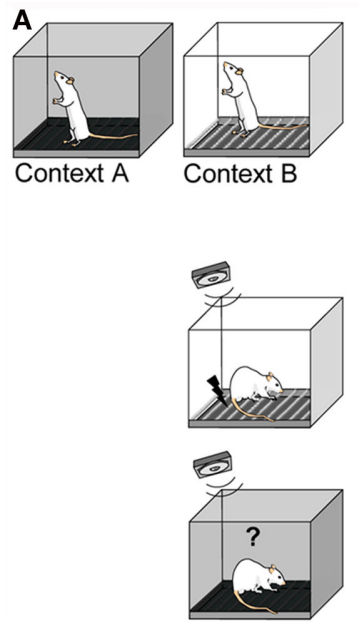

FIGURE 1 | Experimental design. (A) Diagram showing experimental protocol. Animals were pre-exposed to Context A and B for $15 \mathrm{~min}$ the day before conditioning. Context $A$ and context $B$ were use as training and testing
Exposure to each box $(15 \mathrm{~m})$

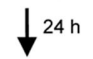

Infusion of vehicle/muscimol

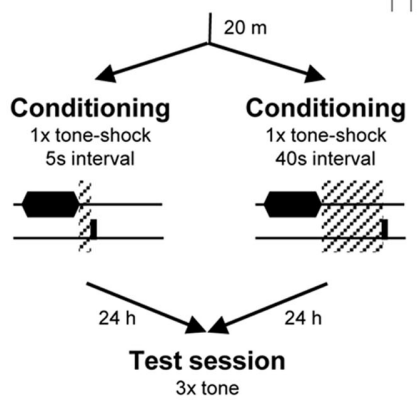

\section{B Amygdala}

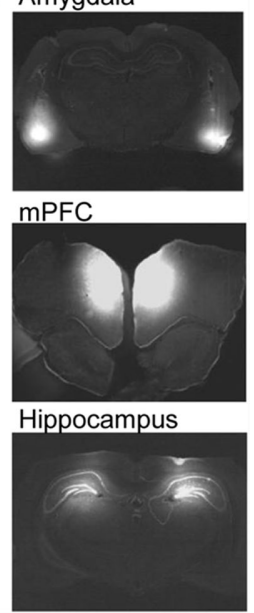

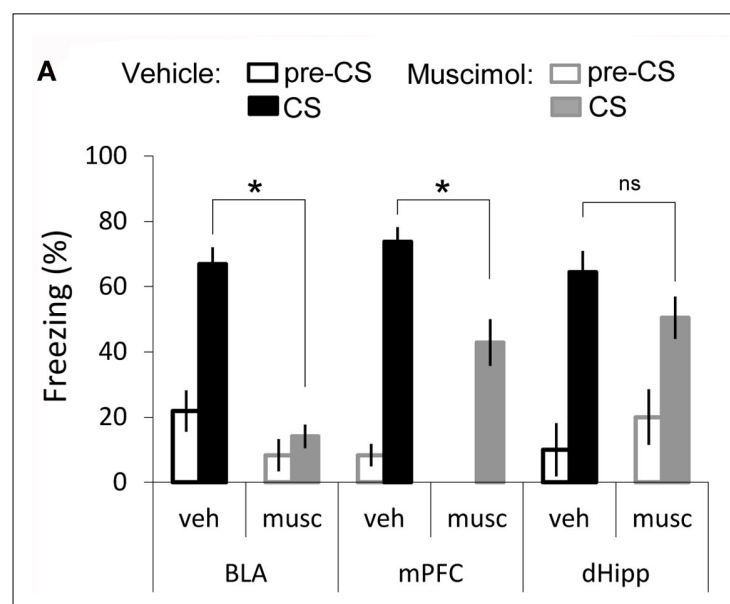

FIGURE 2 | Recruitment of amygdala, medial prefrontal-cortex but not dorsal-hippocampus in short tFC. (A) Bar graph showing percent time spent freezing during the tone CS, averaged across the three tones of the test session and across animals (average \pm SEM) and during the first pre-CS period, in vehicle and muscimol infusion groups. environments in a counterbalanced fashion. (B) Photomicrographs showing example coronal sections of the brain of rats infused with fluorescently labeled muscimol targeted to BLA, mPFC, and dorsal-hippocampus.
mPFC: veh $=35.7 \pm 4.9$, mus $=13.3 \pm 4.0$; dorsal-hippocampus: veh $=30.7 \pm 6.1$, mus $=11.3 \pm 3.1$ ). These differences, however, were not statistically significant.

\section{EXPERIMENT 2}

Role of the amygdala, mPFC, and dorsal-hippocampus in the acquisition of long tFC (tone and shock are separated by $40 \mathrm{~s}$ ).

One of the major goals of this study was to examine if the temporal interval duration between two stimuli could be a factor influencing the neuronal structures that are required for auditory fear conditioning acquisition. Thus, in Experiment 2 the procedures were the same as described in Experiment 1 except for the duration of the interval that separated tone
(B) Cannula placement for both vehicle and muscimol infused rats in each structure: amygdala (BLA) [amyg-mus $(n=9)$; amy-veh $(n=8)]$, medial prefrontal-cortex (mPFC) [mPFC-mus $(n=7)$; mPFC-veh $(n=9)$ ], and dorsal-hippocampus (dHipp) [dHipp-mus $(n=7)$; dHipp-veh $(n=6)$ ]. *Denotes $P<0.017$. 


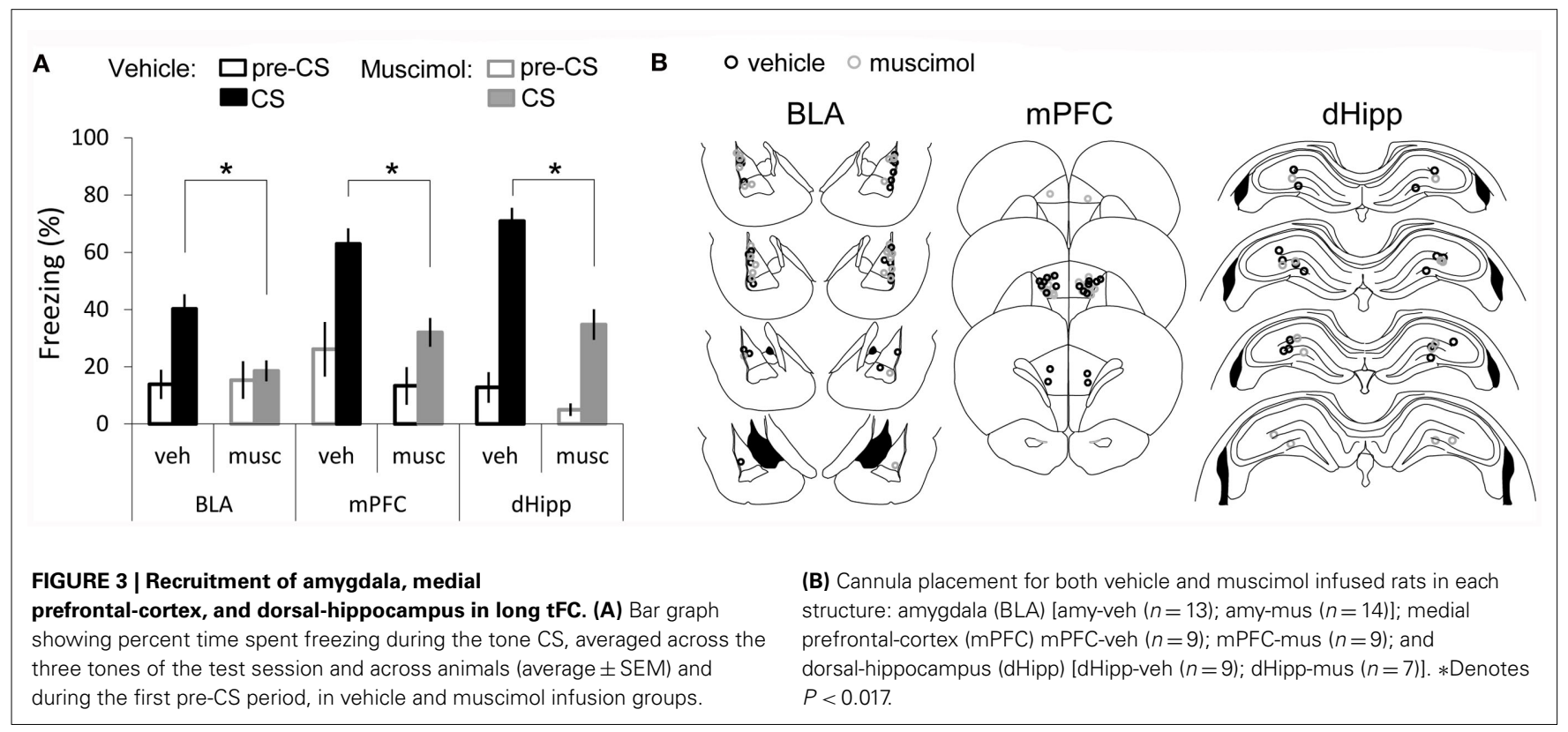

showed significant lower fear of the tone compared to controls $(P=0.009, P=0.003, P<0.001$ respectively; Figure 3). Again to verify that these differences were not due to alterations in baseline fear, we compared pre-CS freezing across all groups and found no significant effect [Kruskal-Wallis: $H_{(5,61)}=2.8, P=0.72$ ] . In addition, as for Experiment 1, we compared post-shock freezing of rats infused with vehicle or muscimol in the amygdala and found no difference $(v e h=25.1 \pm 5.9$, musc $=24.1 \pm 7.2$ ).

In summary, in clear contrast with the short tFC (Experiment 1), all the three areas are critically involved in forming an intact association between tone and shock across a long interval of time. As shown in Figure 2, vehicle infusion into the amygdala seems to decrease freezing to the tone. This could be due to cannula placement, vehicle infusion, or both. Importantly, even though vehicle infusion in amygdala disrupts to some degree freezing to the tone, infusing muscimol had an additional significant effect ruling out the possibility that the deficit observed in muscimol infused rats could be explained solely by the cannula implant and/or injection procedure.

In addition, we measured freezing to the training environment and found that contextual fear learning with the long tFC protocol was even less reliable than the one observed in the short tFC protocol. Still, similarly to results of the short tFC protocol, in this experiment we observed that muscimol infused rats tended to show lower levels of freezing (amygdala: veh $=25.9 \pm 3.8$, mus $=7.4 \pm 2.7$; mPFC: veh $=19.8 \pm 5.0$, mus $=13.9 \pm 4.0$; dorsal-hippocampus: veh $=22.1 \pm 3.8$, mus $=14.5 \pm 3.2$ ).

\section{EXPERIMENT 3}

Role of the mPFC in the acquisition of single-trial dFC (tone and shock are separated by $0 \mathrm{~s}$ ).

Results presented in Experiments 1 and 2 suggest that the mPFC is required for tFC acquisition regardless the length of the trace interval. Previous studies using conditioning protocols consisting of multiple trials of co-terminating tone-shock pairings (dFC) have shown that under such conditions associative fear learning is independent of the mPFC (Morgan and LeDoux, 1995; Corcoran and Quirk, 2007). However, our protocol consisted of a single training trial raising the possibility that this structure was involved in both the short and the long tFC because it is required for singletrial learning and not because tone and shock were separated in time.

Similarly, in Experiment 1 and 2 we show that the amygdala inactivation impairs freezing when either a short or a long interval is imposed between tone and shock. Importantly, unlike the mPFC, the amygdala has been consistently implicated in fear associative learning when no interval is imposed between the stimuli (Fanselow and LeDoux, 1999; LeDoux, 2003; Rumpel et al., 2005; Han et al., 2007, 2009; Maren, 2008; Pape and Pare, 2010) including for single-trial protocols (Schafe and LeDoux, 2000; Schafe et al., 2000). Finally, we show that the hippocampus is involved in the long tFC, but not in the short tFC. In addition, when either multiple or a single-trials are employed for conditioning, $\mathrm{dFC}$ acquisition occur independently of the dorsal-hippocampus (Kim and Fanselow, 1992; Phillips and LeDoux, 1992; Misane et al., 2005; Esclassan et al., 2009). Therefore, only the role of mPFC in delay auditory fear conditioning ( $0 \mathrm{~s}$ interval condition) when a single-trial is used for training, remained to be tested. Thus, in Experiment 3, rats received either muscimol $(n=12)$ or vehicle infusions $(n=8)$ into the mPFC prior to a single-trial of dFC, where tone and shock co-terminate (Figure 4). A $t$-test testing for differences between treatments showed that there was no significant difference in the levels of freezing displayed to the tone between rats from the muscimol and vehicle groups $(P=0.202)$. Finally, we found no difference between baseline freezing (MannWhitney $U$-test: $U=36, P=0.61)$. This demonstrates that the $\mathrm{mPFC}$ is not necessary for single-trial auditory fear learning when the two stimuli overlap in time, thus confirming a role of $\mathrm{mPFC}$ in the association of temporally discontiguous stimuli. 


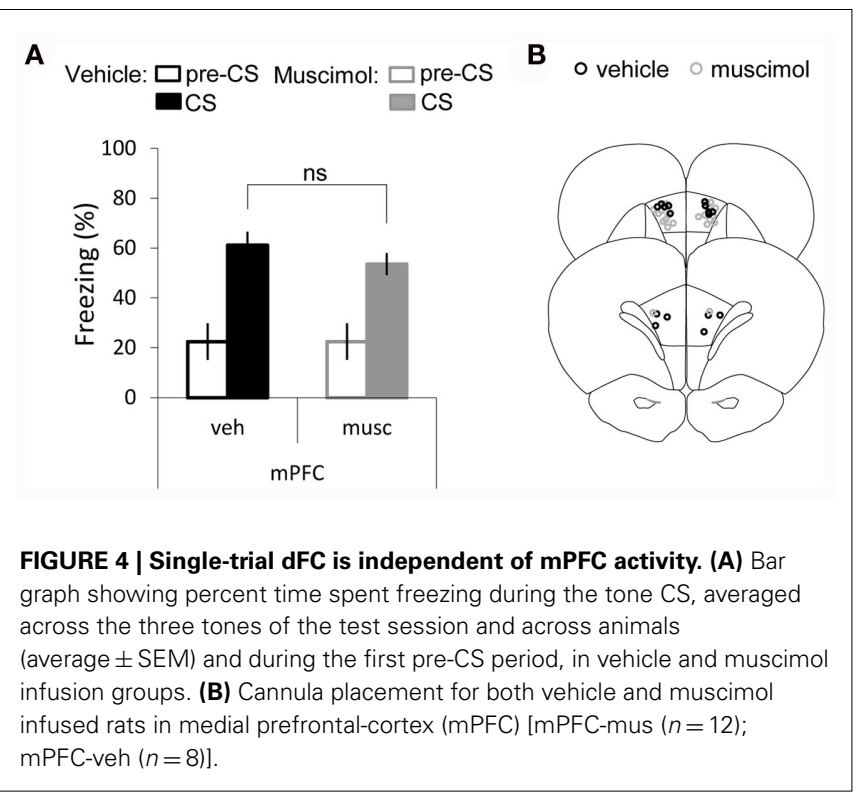

\section{DISCUSSION}

The present experiments show that the amygdala plays an important role in $\mathrm{tFC}$ acquisition and that its involvement is independent of the length of the trace interval. Additionally, our findings implicate the mPFC in both the short and long tFC. Finally, our results further support previous studies on the requirement for dorsal-hippocampus in tFC only when long intervals are used.

The finding that the amygdala is critically involved in trace conditioning regardless of the length of the trace interval is consistent with a vast literature showing that the amygdala plays a crucial role in all or most forms of fear learning tested (Fanselow and LeDoux, 1999; LeDoux, 2003; Maren, 2008; Pape and Pare, 2010). However, a recent report shows that $\mathrm{tFC}$ can be independent of activity in the amygdala (Raybuck and Lattal, 2011). There were a few differences that may explain the discrepancy between our results and those reported by Raybuck and Lattal (2011). In their study mice were used as subjects, whereas in the present study rats were used. Nevertheless, we believe that the difference in the results is unlikely to be due to a difference in how the two species acquire tFC, since the neural mechanism of fear learning seem to be conserved in mammals (Fanselow and LeDoux, 1999; LeDoux, 2003; Maren, 2008; Pape and Pare, 2010). On the other hand differences in how fear of the tone was tested might explain the discrepancy of the results. In our study the length of the tone was the same during training and testing, i.e., 20 s, however in Raybuck's study mice were trained with a 30-s tone but tested to a 3-m tone. Although we do not have a clear explanation as to why the difference in protocol yields a different outcome regarding the recruitment of amygdala, differences in the behavioral protocols commonly lead to discrepancies in the results (Phillips and LeDoux, 1992; Maren et al., 1997). In this study we cannot rule out the possibility that the effects of amygdala inactivation are due to state dependent learning, since rats learn in one state (muscimol in amygdala) and tested in a different state (drug free). Since disruption of activity in basolateral amygdala complex affects the expression of freezing to the tone, state dependentlearning cannot be put to test as it would require performing a double infusion one before the training session and another before test session. Finally, it is still possible that the amygdala is involved in $\mathrm{tFC}$ during the consolidation process and that the effect of muscimol injection prior to training results from lingering muscimol in the amygdala for a period of time after conditioning. However, it has previously been shown that posttraining injections of muscimol into the amygdala do not affect delay auditory fear conditioning, in contrast with the effects shown in inhibitory avoidance (Wilensky et al., 1999, 2000). Whether the involvement of amygdala activity during the consolidation period depends on the interval between tone and shock remains to be tested.

Overall this study demonstrates that, although rats trained with two different $\mathrm{tFC}$ protocols apparently learned the same, i.e., they expressed fear responses to a previously innocuous tone, the association occurs through distinct neuronal circuits. The differential recruitment of brain structures suggests that distinct strategies are engaged to solve the same problem. Hebbian plasticity, thought to underlie associative learning, depends on correlated activity between connected neurons, implying that activation by the tone and shock would have to co-occur within a temporal window of hundreds of milliseconds (Blair et al., 2001). In dFC, where tone and shock overlap in time, co-activation by tone and shock does not pose a problem, while in $\mathrm{tFC}$ the activity driven by the tone presentation must be somehow sustained after the tone offset for the duration of the trace interval. An extensive literature implicates the mPFC in working-memory (Goldman-Rakic, 1995; Fuster, 2001), consistent with its involvement in tasks such as, trace eye blink conditioning (Kronforst-Collins and Disterhoft, 1998; Weible et al., 2000; Kalmbach et al., 2009), delayed choice tasks (Fuster and Alexander, 1971; Fuster et al., 2000), or interval timing (Dietrich and Allen, 1998; Kim et al., 2009). Therefore, a possible way to bridge the tone and shock across a short interval would be by engaging a working-memory like strategy with $\mathrm{MPFC}$ withholding the memory of the tone. Since the mPFC, in particular the pre-limbic cortex sends direct excitatory projections to the basal and lateral amygdala (McDonald, 1998; Hoover and Vertes, 2007), activity in the mPFC during the trace interval could directly drive amygdala neurons until shock delivery. In this case information about the tone at the time of shock delivery would reach the amygdala from $\mathrm{mPFC}$ projections, rather than from projections of auditory sensory areas which predominate during delay conditioning (tone and shock co-terminating). Future experiments are needed to test this hypothesis directly. In our experimental conditions, the mPFC underlies tone-shock association across a short period of time $(5 \mathrm{~s})$, nonetheless $\mathrm{mPFC}$ activity is not sufficient when the temporal gap between stimuli increases. The additional requirement in long tFC of the dorsal-hippocampus, known to be necessary for the encoding of personally experienced episodes (i.e., associating events to the place and time where they occurred; Moita et al., 2003; Eichenbaum, 2004), suggests that an episodic memory like strategy could be employed for tone-shock association across longer intervals. Thus, associating tone and shock could be achieved by encoding these stimuli as part of the same episode (training session) so that subsequent presentation of the tone alone is enough for reactivating the memory of the aversive 
conditioning session and lead to the display of fear responses. The requirement of both hippocampal and $\mathrm{mPFC}$ activity during long $\mathrm{tFC}$, raises the question of whether these two regions act in concert or independently. In the first case it is possible that activity in mPFC is important for normal episodic memory formation in the hippocampus. Indeed it has recently been shown that mPFC plays a role in contextual fear conditioning (Gilmartin and Helmstetter, 2010), which is a hippocampal-dependent task (Sanders et al., 2003), and functional disconnections between mPFC and hippocampus disrupt a spatial task that requires memory of previous trials when long inter-trial intervals were used (Lee and Kesner, 2003). In the later case, the hippocampus and the mPFC would drive cells in amygdala independently and each would contribute to the formation of the association at different sub-sets of synapses or cells, all of which would contribute to a memory of a tone-shock association strong enough to elicit freezing to the tone during the test session. Although further studies are necessary to elucidate how the brain works to associate events that are separated in time our results clearly indicate that the need to link stimuli across increasing time intervals requires more cognitive processing thus recruiting additional brain structures.

Importantly, rats learn to fear the tone after a single pairing with shock. Nonetheless, much of what is known about the neuronal mechanisms underlying associative learning comes from studies where animals are extensively trained. The neuronal mechanisms underlying one-trial learning are likely to be divergent from the ones engaged in processing repetitive events (Miyashita et al., 2009). For instance, based on multiple-trial learning it is predicted that the stronger the learning about the discreet tone $\mathrm{CS}$, the weaker the association with the background context and vice versa (Gallistel and Gibbon, 2000). It follows that when longer intervals separate tone and shock, a weaker tone-shock association is expected and concomitantly a stronger context-shock association is established. However, in our data set there are only small differences in both the tone-shock or context-shock association between short and long tFC. Furthermore, it is known that in multiple-trial conditioning the learning rate is in part determined by the ratio between the inter-stimulus interval and the inter-trial interval (Gallistel and Gibbon, 2000; Balsam and Gallistel, 2009). In essence this means that it is not the absolute length of the interval between the conditioned stimulus, CS (in our case the tone), and the unconditioned stimulus, US (in our case the footshock), but rather the relationship between this same interval and the size of the interval between trials, which affects the formation of the CS-US association. One implication is that interval timing is an important process in multiple-trial but not a single-trial associative learning. How the absolute length of time that elapses between stimuli affects learning remains largely unexplored. The present study presents evidence that keeping information about an event over different timescales may greatly impact the brain areas involved in such process. We believe that using single-trial learning tasks will be instrumental in understanding how the brain deals with information at different time scales. Finally, in the natural environment, animals often learn promptly since a single failure may be fatal. Therefore, additional studies on the neuronal basis of single-trial learning may reveal novel mechanisms by which animals learn to make inferences about the temporal structure of the natural world.
In this study we have identified how the temporal features of tFC change the nodes of the neural circuit underlying this form of learning. In the future it would be important to investigate how activity in these nodes might explain how information about the tone is kept in time and how the connections between the three brain regions studied contribute to this process.

\section{MATERIALS AND METHODS SUBJECTS}

Naive male Sprague Dawley rats (300-450 g) were obtained from a commercial supplier (Harlan). After arrival animals were single housed in Plexiglas top filtered cages and maintained on a 12-h light/dark cycle (lights off at 7:00 p.m.) with ad libitum access to food and water. Rats were acclimated for at least 1 week before experimental manipulation and all animals were handled for a few days before each experiment. All behavioral and surgical procedures were performed during the light phase of the cycle. The Instituto Gulbenkian de Ciência follows the European Guidelines of animal care. The use of vertebrate animals in research in Portugal complies with the European Directive 86/609/EEC of the European Council.

\section{SURGERY}

Rats were anesthetized with sodium pentobarbital ( $65 \mathrm{mg} / \mathrm{kg}$, i.p.) and injected with atropine $(0.4 \mathrm{mg} / \mathrm{kg}$, i.p. $)$ to prevent airway obstruction. After head shaving, rats were place in a stereotaxic instrument with the head secure by non-puncture ear bars. In all surgeries head position was adjusted to place bregma and lambda in the same horizontal plane. Under aseptic conditions, the scalp was incised and skull surface cleared. Using stereotaxic coordinates derived from Paxinos and Watson (2007) two small holes were drilled for bilateral guide cannula (Plastics One) implant. In each animal one of the three structures (Amygdala, mPFC, or dorsal-hippocampus) was targeted. The coordinates were as follow.

\section{Amygdala (BLA)}

Due to its more ventral location and in order to increase the probability of correct cannula placement two reference points (bregma and interaural zero) were used. Prior to cannula implantation, the three coordinates (anterior-posterior, lateral, and ventral) were determined for the two reference points. The final coordinates for 24 gage cannula position were a mean value obtained from the values relative to bregma and to interaural zero. Bregma $3.3 \mathrm{~mm}$ posterior; $5.3 \mathrm{~mm}$ lateral; $-8.2 \mathrm{~mm}$ ventral to skull surface; Interaural zero - $5.7 \mathrm{~mm}$ anterior; $5.3 \mathrm{~mm}$ lateral; $1.8 \mathrm{~mm}$ dorsal.

\section{mPFC}

Twenty-six gage double guide cannula implanted $3.2 \mathrm{~mm}$ anterior to bregma; $0.75 \mathrm{~mm}$ lateral to midline; $4 \mathrm{~mm}$ ventral to skull surface.

\section{Dorsal-hippocampus}

Twenty-four gage guide cannula implanted $3.8 \mathrm{~mm}$ posterior to bregma; $2.5 \mathrm{~mm}$ lateral to midline; $3.8 \mathrm{~mm}$ ventral to skull surface.

The cannulae were secured in place with anchoring screws (Plastics One) and dental cement (TAB 2000-Henry Schein, Inc.). 
Dummy cannulae ( $1 \mathrm{~mm}$ projection) were inserted into each cannula to prevent clogging. Following surgery rats received a single shot of the analgesic buprenorphine $(0.02 \mathrm{mg} / \mathrm{kg})$ and were kept warm and under observation until recovery from anesthesia. All subjects were housed individually and allowed at least 7 days recovery from surgery before any subsequent behavioral procedure.

\section{APPARATUS}

Two distinct environments (A and B) were used in this study. They were located in the same procedure room and were used in a counterbalanced manner (i.e., the animals conditioned in A were tested in $B$ and vice versa). Both consisted of one conditioning chamber (model H10-11R-TC, Coulbourn Instruments) inside a high sound isolation chamber (Action automation and controls, Inc.). During training, both chambers had a shock floor of metal bars (model H10-11R-TC-SF, Coulbourn Instruments). For the tone test sessions, a painted acrylic plate covered the chamber's floor. To minimize generalization between the two environments, several features of the environments differed. In box A, the ceiling and all four sidewalls were made of clear Plexiglas and the walls of sound attenuating cubicle were white. The house light was in the middle-top of the left wall and the speaker was placed outside the chamber, behind the right wall. In Box B, the two sidewalls were made of polished sheet metal. The walls of the sound attenuating cubicle were black. The house light placed in the top-back corner of the right wall and the speaker was behind the left wall. The boxes were cleaned with two cleaning fluids with distinct odors (lavender and natural soap odor). The tones were produced by a sound generator (RM1, Tucker-Davis Technologies) and delivered through a horn tweeter (model TL16H8OHM, VISATON). The sound was calibrated using a Brüel and Kjaer microphone (type 4189) and sound analyzer (hand held analyzer type 2250). A precision programmable shocker (model H13-16, Coulbourn Instruments) delivered the foot-shocks. The rats' behavior was tracked by a video camera mounted on the ceiling of each attenuating cubicle. A surveillance video acquisition system was used to record and store all videos in hard disk for posterior manual off-line scoring of freezing behavior. Scoring was performed by an experimenter blind to the treatment of the animals.

\section{BEHAVIORAL PROCEDURES}

On the first experimental day rats were exposed to the training and testing boxes for $15 \mathrm{~min}$ each. For each rat exposure to the two boxes was separated by an interval ranging from 5 to $6 \mathrm{~h}$. The sessions aimed at improving discrimination between the two environments and thus decreasing pre-CS freezing during the tone test session. The next day, rats were conditioned under one of three training protocols, $\mathrm{dFC}$, short, or long $\mathrm{tFC}$. In order to temporarily inactivate the different brain areas, $5 \mathrm{mg}$ of muscimol, a $\mathrm{GABA}_{\mathrm{A}}$ receptor agonist, was dissolved into $5 \mathrm{ml}$ of $0.01 \mathrm{M}$ phosphatebuffered $0.9 \%$ saline (PBS) for a final concentration of $1.6 \mathrm{mM}$. The dummy cannulae were removed and an injection cannulae extending $2 \mathrm{~mm}$ below the tip of the guide cannula was inserted ( 31 gage for amygdala and dorsal-hippocampus, 33 gage for mPFC), a procedure to which rats were habituated for a few days prior to training. The injection cannulae filled with mineral oil and connected to a 1- $\mu l$ syringe (Hamilton), were loaded with muscimol or vehicle through backfilling. Rats received either an infusion of PBS (vehicle) or muscimol (dorsal-hippocampus $0.4 \mu \mathrm{l}$; mPFC $0.3 \mu \mathrm{l}$; amygdala $0.3 \mu \mathrm{l}$ ) at a rate of $0.25 \mu \mathrm{l} / \mathrm{min}$ using an infusion pump (Harvard Apparatus). The injection cannulae were left in place for an additional $90 \mathrm{~s}$ to achieve a proper diffusion of the drug or vehicle solution from the tips. Twenty minutes following infusion animals were subjected to a fear conditioning. In all protocols animals were placed inside the training box and $10 \mathrm{~min}$ later they received one tone presentation $(20 \mathrm{~s} ; 5 \mathrm{kHz} ; 70 \mathrm{~dB})$ followed by a foot-shock $(1.5 \mathrm{~mA} ; 1.5 \mathrm{~s})$. The interval between tone and shock was $5 \mathrm{~s}$ for "short tFC" (short tFC) or $40 \mathrm{~s}$ for "long tFC" (long $\mathrm{tFC}$ ) or $0 \mathrm{~s}$ for the dFC. Five minutes after shock delivery rats were removed from the box and returned to their home cage. Twenty-four hours after training all rats were subjected to a tone test session. Testing took place in a different box and consisted of a 5-min baseline period followed by three tone presentations (20 s duration each) delivered with an average inter-trial interval of $180 \mathrm{~s}$ (ranging from 170 to $190 \mathrm{~s}$ ). At the end of the session animals were returned to their home cages.

\section{HISTOLOGY}

Upon conclusion of all behavioral procedures, the animals were deeply anesthetized with an overdose of sodium pentobarbital. In order to have a general estimation of the spread of the drug' fluorescent Muscimol (BODIPY TMR-X muscimol; Invitrogen) was injected targeting the structure just after anesthesia induction. Fluorescently labeled muscimol was not use for the infusions during the experiment (prior to training) for two reasons: (1) since rats were tested for fear of the tone and of the context on two consecutive days after training, by the time the animals were sacrificed no fluorescence would be detectable due to drug washout; (2) when testing BODIPY dissolved in PBS as control vehicle solution we found the behavior of rats to be disrupted, hence we are concerned that BODIPY may be toxic. The drug infusion procedure was identical to the one performed before training (see above). Following infusion, rats were decapitated and the brains removed to be stored at $-4^{\circ} \mathrm{C}$ in a $30 \%$ sucrose/paraformaldehyde postfix solution until they sank (2-3 days). The brains were frozen and $40 \mu \mathrm{m}$ thick coronal sections covering the whole extent of the structure of interest (amygdala, mPFC, or dorsal-hippocampus) were cut on a cryostat. Every second section was collected on coated slides. Sections were then analyzed and photographed using a fluorescence stereoscope (Zeiss stereo Lumar), for verification of drug dispersion area and injector cannulae tip placement. The behavioral data for rats with either one or both tip cannulae outside of the target area were excluded.

\section{STATISTICAL ANALYSIS}

Freezing was used as the index of conditioned fear. An episode of freezing was defined as a period in which there was total immobility except for the respiratory related movement. The duration of time spent freezing was scored at specified time periods during the tone test session: $20 \mathrm{~s}$ immediately before the first tone presentation (baseline freezing) and during each one of the three $20 \mathrm{~s}$ tones. In addition, post-shock freezing was measured during $60 \mathrm{~s}$ following the foot-shock of the training session (for amygdala 
implanted rats). Animals which displayed abnormal baseline freezing, defined as values of freezing during the baseline period above $\mathrm{Q}_{3}+1.5 \times\left(\mathrm{Q}_{3}-\mathrm{Q}_{1}\right)$, corresponded to freezing score above 71\%, were excluded. In total, seven rats were excluded: One from the 5-s dorsal-hippocampus muscimol group; one from the delaymPFC-vehicle group; three from the 40-s amygdala vehicle group and two from the 5-s amygdala vehicle group. In addition, 11 rats were excluded after histology for cannulae misplacement. A total of 127 rats were included in this study belonging to 1 of the 14 groups that composed the three experiments.

\section{Experiment 1}

Short tFC protocol (5s) with six groups: amy-veh $(n=8)$; amymus $(n=9)$; mPFC-veh $(n=9)$; mPFC-mus $(n=7)$; dHipp-veh $(n=6)$; dHipp-mus $(n=7)$.

\section{Experiment 2}

Long tFC protocol (40 s) with six groups: amy-veh $(n=13)$; amymus $(n=14)$; mPFC-veh $(n=9)$; mPFC-mus $(n=9)$; dHipp-veh $(n=9)$; dHipp-mus $(n=7)$.

\section{Experiment 3}

dFC protocol ( $0 \mathrm{~s})$ with two groups: mPFC-mus $(n=12)$; mPFCveh $(n=8)$.

In all groups baseline freezing values did not follow a normal distribution (Shapiro-Wilk test), therefore to compare pre-CS freezing across the different group non-parametric test were used (Kruskal-Wallis in the case of short and long tFC and MannWhitney $U$ for the dFC). For each rat, percent freezing during each

\section{REFERENCES}

Baeg, E. H., Kim, Y. B., Jang, J., Kim, H. T., Mook-Jung, I., and Jung, M. W. (2001). Fast spiking and regular spiking neural correlates of fear conditioning in the medial prefrontal cortex of the rat. Cereb. Cortex 11, 441-451.

Balsam, P. D., and Gallistel, C. R. (2009). Temporal maps and informativeness in associative learning. Trends $\mathrm{Neu}$ rosci. 32, 73-78.

Blair, H. T., Schafe, G. E., Bauer, E. P., Rodrigues, S. M., and LeDoux, J. E. (2001). Synaptic plasticity in the lateral amygdala: a cellular hypothesis of fear conditioning. Learn. Mem. 8 , 229-242.

Blair, H. T., Sotres-Bayon, F., Moita, M. A., and Ledoux, J. E. (2005). The lateral amygdala processes the value of conditioned and unconditioned aversive stimuli. Neuroscience 133, 561-569.

Büchel, C., Dolan, R. J., Armony, J. L., and Friston, K. J. (1999). Amygdalahippocampal involvement in human aversive trace conditioning revealed through event-related functional magnetic resonance imaging. J. Neurosci. 19, 10869-10876.

Chowdhury, N., Quinn, J. J., and Fanselow, M. S. (2005). Dorsal hippocampus involvement in trace fear conditioning with long, but not short, trace intervals in mice. Behav. Neurosci. 119, 1396-1402.

Christian, K. M., and Thompson, R. F. (2003). Neural substrates of eye blink conditioning: acquisition and retention. Learn. Mem. 10, 427-455.

Ciocchi, S., Herry, C., Grenier, F., Wolff, S. B., Letzkus, J. J., Vlachos, I., Ehrlich, I., Sprengel, R., Deisseroth, K., Stadler, M. B., Müller, C., and Lüthi, A. (2010). Encoding of conditioned fear in central amygdala inhibitory circuits. Nature 468, 277-282.

Corcoran, K. A., and Quirk, G. J. (2007). Activity in prelimbic cortex is necessary for the expression of learned, but not innate, fears. J. Neurosci. 27, 840-844.

Dietrich, A., and Allen, J. D. (1998). Functional dissociation of the prefrontal cortex and the hippocampus in timing behavior. Behav. Neurosci. 112, 1043-1047.

Eichenbaum, H. (2004). Hippocampus: cognitive processes and neural representations that underlie declarative memory. Neuron 44,109-120.

Esclassan, F., Coutureau, E., Di Scala, G., and Marchand, A. R. (2009). Differential contribution of dorsal and

tone was averaged across the three trials of the tone test session. In all groups but one, the mean values followed a normal distribution. Importantly, all groups met the homoscedasticity criterion. The results for each protocol (short tFC or long tFC) were analyzed by a two-way ANOVA testing for main effect of structures (amygdala or mPFC or dorsal-hippocampus) and treatment (muscimol vs. vehicle). Post hoc comparisons were performed using LSD test Bonferroni corrected for multiple comparisons $(\alpha=0.017)$. For the one group that did not follow a normal distribution [mPFCveh from the short tFC protocol (5s)] we confirmed the result obtained with the parametric analysis using a non-parametric Mann-Whitney $U$-test, which revealed a significant difference between vehicle and muscimol infused rats $(U=12, P=0.039)$. A $t$-test was performed to analyze the differences between treatments for mPFC infusions in Experiment 3. All statistical analysis was performed using the application Statistica version 8 (StatSoft, Inc.).

\section{ACKNOWLEDGMENTS}

We thank R. Antunes, J. Pathon, A. M. Poulos, A. Pereira, S. Rennie, and E. Sucena for helpful discussion and comments on this manuscript. We thank M. Vinhas, and A. Santos for the histology work. This work was supported by Fundação Champalimaud, Instituto Gulbenkian de Ciência, Fundação para a Ciência e Tecnologia, Portugal (PTDC/SAUNEU/71647/2006), and Fundação Bial, Portugal. Marta Guimarãis was supported by Fundação para a Ciência e Tecnologia, Portugal SFRH/BD/28343/2006

ventral hippocampus to trace and delay fear conditioning. Hippocampus 19, 33-44.

Fanselow, M. S., and LeDoux, J. E. (1999). Why we think plasticity underlying Pavlovian fear conditioning occurs in the basolateral amygdala. Neuron 23, 229-232.

Fuster, J. M. (2001). The prefrontal cortex-an update: time is of the essence. Neuron 30, 319-333.

Fuster, J. M., and Alexander, G. E. (1971). Neuron activity related to short-term memory. Science 173, 652-654.

Fuster, J. M., Bodner, M., and Kroger, J. K. (2000). Cross-modal and crosstemporal association in neurons of frontal cortex. Nature 405, 347-351.

Gallistel, C. R., and Gibbon, J. (2000). Time, rate and conditioning. Psychol. Rev. 107, 289-344.

Gilmartin, M. R., and Helmstetter, F. J. (2010). Trace and contextual fear conditioning require neural activity and NMDA receptor-dependent transmission in the medial prefrontal cortex. Learn. Mem. 17, 289-296.

Gilmartin, M. R., and McEchron, M. D. (2005a). Single neurons in the medial prefrontal cortex of the rat exhibit tonic and phasic coding during trace fear conditioning. Behav. Neurosci. 119, 1496-1510.

Gilmartin, M. R., and McEchron, M. D. (2005b). Single neurons in the dentate gyrus and CAl of the hippocampus exhibit inverse patterns of encoding during trace fear conditioning. Behav. Neurosci. 119, 164-179.

Goldman-Rakic, P. S. (1995). Cellular basis of working memory. Neuron 14, 477-485.

Han, J. H., Kushner, S. A., Yiu, A. P., Cole, C. J., Matynia, A., Brown, R. A., Neve, R. L., Guzowski, J. F., Silva, A. J., and Josselyn, S. A. (2007). Neuronal competition and selection during memory formation. Science 316 , 457-460.

Han, J. H., Kushner, S. A., Yiu, A. P., Hsiang, H. L., Buch, T., Waisman, A., Bontempi, B., Neve, R. L., Frankland, P. W., and Josselyn, S. A. (2009). Selective erasure of a fear memory. Science 323, 1492-1496.

Haubensak, W., Kunwar, P. S., Cai, H. Ciocchi, S., Wall, N. R., Ponnusamy, R., Biag, J., Dong, H. W., Deisseroth, K., Callaway, E. M., Fanselow, M. S., Lüthi, A., and Anderson, D. J. (2010). Genetic dissection of an amygdala microcircuit that gates conditioned fear. Nature 468, 270-276. 
Hoover, W. B., and Vertes, R. P. (2007). Anatomical analysis of afferent projections to the medial prefrontal cortex in the rat. Brain Struct. Funct. 212, 149-179.

Huerta, P. T., Sun, L. D., Wilson, M. A., and Tonegawa, S. (2000). Formation of temporal memory requires NMDA receptors within CA1 pyramidal neurons. Neuron 25, 473-480.

Johansen, J. P., Tarpley, J. W., LeDoux, J. E., and Blair, H. T. (2010). Neural substrates for expectationmodulated fear learning in the amygdala and periaqueductal gray. Nat. Neurosci. 13, 979-986.

Kalmbach, B. E., Ohyama, T., Kreider, J. C., Riusech, F., and Mauk, M. D. (2009). Interactions between prefrontal cortex and cerebellum revealed by trace eyelid conditioning. Learn. Mem. 16, 86-95.

Kim, J., Jung, A. H., Byun, J., Jo, S., and Jung, M. W. (2009). Inactivation of medial prefrontal cortex impairs time interval discrimination in rats. Front. Behav. Neurosci. 3:38. doi:10.3389/neuro.08.038.2009

Kim, J. J., and Fanselow, M. S. (1992). Modality-specific retrograde amnesia of fear. Science 256, 675-677.

Kronforst-Collins, M. A., and Disterhoft, J. F. (1998). Lesions of the caudal area of rabbit medial prefrontal cortex impair trace eyeblink conditioning. Neurobiol. Learn. Mem. 69, 147-162.

Kwapis, J. L., Jarome, T. J., Schiff, J. C., and Helmstetter, F. J. (2011). Memory consolidation in both trace and delay fear conditioning is disrupted by intra-amygdala infusion of the protein synthesis inhibitor anisomycin. Learn. Mem. 18, $728-732$.

LeDoux, J. (2003). The emotional brain, fear, and the amygdala. Cell. Mol. Neurobiol. 23, 727-738.

Lee, I., and Kesner, R. P. (2003). Timedependent relationship between the dorsal hippocampus and the prefrontal cortex in spatial memory. $J$. Neurosci. 23, 1517-1523.

Maren, S. (2008). Pavlovian fear conditioning as a behavioral assay for hippocampus and amygdala function: cautions and caveats. Eur. J. Neurosci. 28, 1661-1666.

Maren, S., Aharonov, G., and Fanselow, M. S. (1997). Neurotoxic lesions of the dorsal hippocampus and
Pavlovian fear conditioning in rats. Behav. Brain Res. 88, 261-274.

McDonald, A. J. (1998). Cortical pathways to the mammalian amygdala. Prog. Neurobiol. 55, 257-332.

McEchron, M. D., Bouwmeester, H. Tseng, W., Weiss, C., and Disterhoft, J. F. (1998). Hippocampectomy disrupts auditory trace fear conditioning and contextual fear conditioning in the rat. Hippocampus 8, 638-646.

McEchron, M. D., Tseng, W., and Disterhoft, J. F. (2003). Single neurons in CA1 hippocampus encode trace interval duration during trace heart rate (fear) conditioning in rabbit. $J$. Neurosci. 23, 1535-1547.

Misane, I., Tovote, P., Meyer, M., Spiess, J., Ogren, S. O., and Stiedl, O. (2005). Time-depedent involvement of the dorsal-hippocampus in trace fear conditioning in mice. Hippocampus $15,418-426$.

Miyashita, T., Kubik, S., Haghighi, N., Steward, O., and Guzowski, J. F. (2009). Rapid activation of plasticity-associated gene transcription in hippocampal neurons provides a mechanism for encoding of one-trial experience. J. Neurosci. 29, 898-906.

Moita, M. A. P., Rosis, S., Zhou, Y., LeDoux, J. E., and Blair, H. T. (2003). Hippocampal place cells acquire location-specific responses to conditioned stimulus during auditory fear conditioning. Neuron 37, 485-497.

Morgan, M. A., and LeDoux, J. E. (1995). Differential contribution of dorsal and ventral medial prefrontal cortex to the acquisition and extinction of conditioned fear in rats. Behav. Neurosci. 109, 681-688.

Muller, J., Corodimas, K. P., Fridel, Z., and LeDoux, J. E. (1997). Functional inactivation of the lateral and basal nuclei of the amygdala by muscimol infusion prevents fear conditioning to an explicit conditioned stimulus and to contextual stimuli. Behav. Neurosci. 111, 683-691.

Pape, H., and Pare, D. (2010). Plastic synaptic networks of the amygdala for the acquisition, expression, and extinction of conditioned fear. Physiol. Rev. 90, 419-463.

Paxinos G., and Watson C. (2007). The Rat Brain in Stereotaxic Coordinates, 6th Edn. Amsterdam: Elsevier.
Phillips, R. G., and LeDoux, J. E. (1992). Differential contribution of amygdala and hippocampus to cued and contextual fear conditioning. Behav. Neurosci. 106, 274-285.

Pitkänen, A., Pikkarainen, M., Nurminen, N., and Ylinen, A. (2000). Reciprocal connections between the amygdala and the hippocampal formation, perirhinal cortex, and postrhinal cortex in rat. A review. Ann. N. Y. Acad. Sci. 911, 369-391.

Quinn, J. J., Loya, F., Ma, Q. D., and Fanselow, M. S. (2005). Dorsal hippocampus NMDA receptors differentially mediate trace and contextual fear conditioning. Hippocampus 5, 665-674.

Quinn, J. J., Oommen, S. S., Morrison, G. E., and Fanselow, M. S. (2002). Post-training excitotoxic lesions of the dorsal hippocampus attenuate forward trace, backward trace, and delay fear conditioning in a temporally specific manner. Hippocampus 12, 495-504.

Raybuck, J. D., and Lattal, K. M. (2011). Double dissociation of amygdala and hippocampal contributions to trace and delay fear conditioning. PLoS ONE 6, e15982. doi:10.1371/journal.pone.0015982

Rumpel, S., LeDoux, J. E., Zador, A. and Malinow, R. (2005). Postsynaptic receptor trafficking underlying a form of associative learning. Science 308, 83-88.

Runyan, J. D., Moore, A. N., and Dash, P. K. (2004). A role for prefrontal cortex in memory storage for trace fear conditioning. J. Neurosci. 24 1288-1295.

Sanders, M. J., Wiltgen, B. J., and Fanselow, M. S. (2003). The place of the hippocampus in fear conditioning. Eur. J. Pharmacol. 463, 217-223.

Schafe, G. E., Atkins, C. M., Swank, M. W., Bauer, E. P., Sweatt, J. D., and LeDoux, J. E. (2000). Activation of ERK/MAP kinase in the amygdala is required for memory consolidation of pavlovian fear conditioning. J. Neurosci. 20, 8177-8187.

Schafe, G. E., and LeDoux, J. E. (2000). Memory consolidation of auditory pavlovian fear conditioning requires protein synthesis and protein kinase A in the amygdala. J. Neurosci. 20, RC96.

Stehberg, J., and Simon, F. (2011). Involvement of the insular cortex in retention of conditioned taste aversion is not time dependent. Neurobiol. Learn. Mem. 95, 14-18.

Stiedl, O., and Spiess, J. (1997). Effect of tone-dependent fear conditioning on heart rate and behavior of C57BL/6N mice. Behav. Neurosci. 111,703-711.

Weible, A. P., McEchron, M. D. and Disterhoft, J. F. (2000). Cortical involvement in acquisition and extinction of trace eyeblink conditioning. Behav. Neurosci. 114, 1058-1067.

Welzl, H., D'Adamo, P., and Lipp, H. P. (2001). Conditioned taste aversion as a learning and memory paradigm. Behav. Brain Res. 125, 205-213.

Wilensky, A. E., Schafe, G. E., and LeDoux, J. E. (1999). Functional inactivation of the amygdala before but not after auditory fear conditioning prevents memory formation. J. Neurosci. 19, RC48.

Wilensky, A. E., Schafe, G. E., and LeDoux, J. E. (2000). The amygdala modulates memory consolidation of fear-motivated inhibitory avoidance learning but not classical fear conditioning. J. Neurosci. 20, 7059-7066.

Woodruff-Pak, D. S., and Disterhoft, J. F. (2008). Where is the trace in trace conditioning? Trends Neurosci. 31, 105-112.

Conflict of Interest Statement: The authors declare that the research was conducted in the absence of any commercial or financial relationships that could be construed as a potential conflict of interest.

Received: 04 July 2011; accepted: 13 December 2011; published online: 27 December 2011.

Citation: Guimarãis $M$, Gregório $A$, Cruz A, Guyon $N$ and Moita MA (2011) Time determines the neural circuit underlying associative fear learning. Front. Behav. Neurosci. 5:89. doi: 10.3389/fnbeh.2011.00089 Copyright () 2011 Guimarãis, Gregório, Cruz, Guyon and Moita. This is an open-access article distributed under the terms of the Creative Commons Attribution Non Commercial License, which permits non-commercial use, distribution, and reproduction in other forums, provided the original authors and source are credited. 